\title{
Disposição em aterros controlados de resíduos sólidos industriais não-inertes: avaliação dos componentes tóxicos e implicações para o ambiente e para a saúde humana
}

\author{
Non-inert industrial solid waste disposal in landfill \\ dumps: evaluation of toxicity and implications \\ for the environment and human health
}

Cristina L. S. Sisinno 1,2

\footnotetext{
${ }^{1}$ Centro de Tecnologia Ambiental, Federação das Indústrias do Estado do Rio de Janeiro. Rua Morais e Silva 53, Rio de Janeiro, $R J$ 20271-030, Brasil. 2 Centro de Estudos de Saúde do Trabalhador e Ecologia Humana, Escola Nacional de Saúde Pública, Fundação Oswaldo Cruz. Rua Leopoldo Bulhões 1480, Rio de Janeiro, $R J$ 21041-210, Brasil. sisinno@ensp.fiocruz.br
}

\begin{abstract}
According to Brazilian recommended technical procedures (mainly NBR 10,004), solid waste must be previously classified in order to be disposed of adequately. Non-inert industrial solid waste is being dumped in landfill areas, most of which operate inefficiently and are located near the population and important ecosystems. In order to evaluate the potential toxicity of solid waste produced by various types of industries, 21 samples were analyzed according to NBR 10,004 procedures. Of these, 18 were classified as non-inert solid waste. The main substances contributing to the classification of these samples as non-inert waste were: aluminum, iron, manganese, phenol, and surfactants. Aluminum, manganese, and phenol are the main toxicologically relevant substances for human and environmental health because they can alter the quality of groundwater situated under solid waste disposal areas.

Key words Solid Wastes; Waste Classification; Sanitary Landfill

Resumo Para que um resíduo sólido seja disposto adequadamente, é necessário classificá-lo segundo as Normas Técnicas Brasileiras, cuja principal é a NBR 10.004. Resíduos sólidos industriais não-inertes normalmente têm sido encaminhados para disposição final em aterros controlados, que em sua maioria não operam de forma eficiente, além de estarem geralmente localizados próximos a núcleos populacionais e ecossistemas importantes. A fim de avaliar a toxicidade potencial dos resíduos produzidos em indústrias de diferentes segmentos, 21 amostras foram analisadas de acordo com as orientações descritas na NBR 10.004. Das amostras estudadas, 18 foram classificadas como resíduos não-inertes. Os principais parâmetros que contribuíram para a classificação das amostras em resíduos não-inertes foram: alumínio, ferro, manganês, fenol e surfactantes. Destes, o alumínio, o manganês e o fenol são as substâncias de maior interesse toxicológico para a saúde humana e ambiental, uma vez que podem alterar a qualidade das águas subterrâneas localizadas nas áreas de disposição de resíduos.
\end{abstract}

Palavras-chave Resíduos Sólidos; Classificação de Resíduos; Aterro Sanitário 


\section{Introdução}

Para que um resíduo tenha destino adequado, é necessário que ele seja classificado de acordo com as normas brasileiras. A NBR 10.004 Classificação de resíduos (ABNT, 1987c) classifica os resíduos em três classes: classe I - perigosos; classe II - não-inertes; classe III - inertes. Essa classificação baseia-se na presença de certas substâncias perigosas, relacionadas na norma, e em testes laboratoriais complementares, nos quais vários parâmetros químicos são analisados nos extratos lixiviados e solubilizados dos resíduos.

Apesar de a NBR 10.004 ser baseada em procedimentos americanos, relacionados no Code of Federal Registry - Title 40 (CFR 40) - Protection of Environment (USA, 1994), a classificação dos resíduos sólidos em três classes é peculiar à norma brasileira, pois o CFR 40 orienta para a classificação dos resíduos apenas em perigosos e não-perigosos, sem mencionar o teste de solubilização dos resíduos, que é o principal responsável pela classificação dos resíduos não-inertes e inertes segundo a norma brasileira.

O processo produtivo, na grande maioria das vezes, tem como conseqüência a geração de resíduos que precisam de tratamento e destino adequados, uma vez que diversas substâncias bastante comuns nos resíduos industriais são tóxicas e algumas têm a capacidade de bioacumulação nos seres vivos, podendo entrar na cadeia alimentar e chegar até o homem.

A realidade vivida pelo setor industrial no Brasil é bastante peculiar. Apesar de o gerador ser o responsável pelo destino de seus resíduos, a escassez de informações e de alternativas disponíveis para esse fim e a carência de pessoal especializado fazem com que algumas indústrias dispensem pouca ou nenhuma atenção a tal responsabilidade. Esse descaso muitas vezes é motivado pela deficiência na fiscalização e na crença de que o tratamento ou destino adequado dos resíduos acarretará altos custos para as empresas.

As indústrias localizadas no Estado do Rio de Janeiro produzem toneladas de resíduos que muitas vezes têm destino desconhecido ou são despejados, sem autorização dos órgãos competentes, em vazadouros (muitos dos quais clandestinos) ou cursos d'água. Entretanto, do total de aproximadamente 500.000 toneladas de resíduos industriais produzidos mensalmente no estado pelas indústrias inventariadas pelo órgão de fiscalização ambiental do Estado do Rio de Janeiro, a Fundação Estadual de Engenharia do Meio Ambiente (FEEMA), a maior parte constitui-se de resíduos não-inertes (classe II), seguido de resíduos inertes (classe III) e, por fim, de resíduos perigosos (classe I) (FEEMA, 2000).

As principais formas de tratamento e destinação dos resíduos industriais produzidos no Estado do Rio de Janeiro são: reciclagem, aterro municipal, co-processamento, aterro industrial, estocagem, incineração, incorporação, fertilização ou landfarming e aterro de terceiros. Com relação aos resíduos não-inertes, porém, as principais formas de tratamento e destino incluem a reciclagem, a estocagem na própria indústria e o despejo em aterros municipais (FEEMA, 2000).

Como o Estado do Rio de Janeiro não possui aterros para resíduos industriais não-inertes, verifica-se por meio dos dados inventariados pela FEEMA que muitos resíduos dessa classe estão sendo depositados em aterros municipais (FEEMA, 2000). No entanto, vários dos aterros localizados no Estado do Rio de Janeiro não operam de forma eficiente, transformando-se em fontes potenciais de contaminação ambiental e de riscos à saúde humana (FEEMA, 1998).

Muitas dessas áreas estão inadequadamente localizadas nas proximidades de cursos d'água, núcleos populacionais e ecossistemas de grande interesse ecológico, como é o caso do aterro de Gramacho, situado na região metropolitana do Rio de Janeiro às margens da Baía de Guanabara, em área de manguezais (COMLURB, 1993), e o aterro controlado do Morro do Céu (Niterói, Rio de Janeiro), instalado em área de florestas secundárias e nascentes (Sisinno \& Oliveira, 2000), cujo potencial de contaminação ambiental tem sido avaliado em alguns estudos, como os de Pereira Netto et al. (2002) e Sisinno \& Moreira (1996).

\section{Materiais e métodos}

Vinte e uma amostras de resíduos provenientes de indústrias de diferentes segmentos (químico, petroquímico, de beneficiamento de minerais, metalúrgico, de alimentos e de bebidas), localizadas no Estado do Rio de Janeiro, foram analisadas de acordo com a NBR 10.004. Muitas dessas indústrias tinham em comum o interesse na implantação das normas do sistema de gestão ambiental (série ISO 14.000) e estavam preocupadas em classificar seus resíduos para destiná-los de forma adequada, já que esta é uma das exigências para a obtenção desse tipo de certificação ambiental. O interesse na implantação de um sistema de gestão ambiental 
está relacionado a uma série de benefícios potenciais, como a manutenção de boas relações com o público/comunidade, o fortalecimento da imagem e a redução de incidentes que impliquem responsabilidade civil, etc. (ABNT, 1996).

As amostras foram coletadas conforme a metodologia recomendada pela NBR 10.007 amostragem de resíduos (ABNT, 1987a) e tratadas de acordo com as metodologias estabelecidas pela Associação Brasileira de Normas Técnicas (ABNT) para os testes de lixiviação (NBR 10.005) e solubilização (NBR 10.006) de resíduos (ABNT, 1987b, 1987d).

Para o processo de lixiviação, $100 \mathrm{~g}$ da massa úmida do resíduo foram misturados a $1.600 \mathrm{~mL}$ de água deionizada. Após o início da agitação da mistura o $\mathrm{pH}$ foi medido e os valores acima de 5 , corrigidos mediante adição de ácido acético 0,5N. A mistura, então, foi agitada por 24 horas e posteriormente filtrada em membrana de fibra de vidro de $0,45 \mu \mathrm{m}$ de porosidade. Esse procedimento simula condições ácidas que favorecem a lixiviação de alguns contaminantes e que podem ocorrer devido à decomposição da matéria orgânica presente nos resíduos urbanos, ao ser misturada com o resíduo industrial em uma área de disposição (ABNT, 1987b).

O processo de solubilização consistiu na mistura de $250 \mathrm{~g}$ da massa seca do resíduo a $1.000 \mathrm{~mL}$ de água deionizada. Essa mistura foi agitada por cinco minutos a baixa velocidade, ficando em repouso, tampada, por sete dias. Após esse período, foi filtrada em membrana de fibra de vidro de $0,45 \mu \mathrm{m}$ de porosidade, originando o extrato solubilizado. Esse procedimento tem por finalidade demonstrar que, uma vez em contato com a água, o resíduo não modificaria a qualidade dos padrões de potabilidade da água (ABNT, 1987d).

Os valores de $\mathrm{pH}$ foram determinados nas amostras brutas dos resíduos pelo método EPA $9045 \mathrm{c}$, no qual $20 \mathrm{~g}$ da amostra bruta foram misturados a $20 \mathrm{~mL}$ de água deionizada. A mistura foi agitada por 5 minutos e, após repouso de 15 minutos, o pH do sobrenadante foi medido (EPA, 1996).

As metodologias utilizadas na determinação dos parâmetros químicos nos extratos dos resíduos lixiviados e solubilizados requeridos pela NBR 10.004 foram baseadas no Standard Methods for the Examination of Water and Wastewater (APHA, 1995).

De acordo com o processo industrial utilizado em cada indústria - e conseqüentemente com a possível presença dos contaminantes em questão nas amostras de resíduos -, foram avaliados os seguintes parâmetros nos resíduos lixiviados: As; $\mathrm{Ba}$; $\mathrm{Cd}$; $\mathrm{Pb}$; $\mathrm{Cr}$ total; $\mathrm{Cr}$ he- xavalente; fluoreto; Hg; Ag e Se. As avaliações foram realizadas em atenção ao Anexo G - Listagem no 7 da NBR 10.004 (ABNT, 1987c).

O mesmo procedimento foi utilizado para os resíduos solubilizados, nos quais foram analisados os seguintes parâmetros: As; $\mathrm{Ba} ; \mathrm{Cd}$; $\mathrm{Pb}$; cianeto; Cr total; fenol; fluoreto; Hg; nitrato; Ag; Se; Al; cloreto; $\mathrm{Cu}$; dureza; Fe; $\mathrm{Mn}$; $\mathrm{Na}$; surfactantes; sulfato e $\mathrm{Zn}$, constantes do Anexo $\mathrm{H}$ - Listagem no 8 da NBR 10.004 (ABNT, 1987c).

Apesar de constarem dos Anexos G e H da NBR 10.004, os contaminantes orgânicos (aldrin; clordano; DDT; dieldrin; endrin; epóxiheptacloro; heptacloro; hexaclorobenzeno; lindano; metoxicloro; pentaclorofenol; toxafeno; 2,4-D; 2,4,5-T; 2,4,5-TP; organofosforados e carbamatos) não foram determinados em nenhuma das amostras estudadas, uma vez que são substâncias de uso restrito e não estavam presentes no processo produtivo das empresas em questão (ABNT, 1987c).

O tratamento preliminar das amostras destinadas à determinação de metais nos extratos solubilizados e lixiviados constou de um ataque com $\mathrm{HNO}_{3}(50 \mathrm{~mL}$ do extrato $+5 \mathrm{~mL}$ de ácido), de acordo com o método EPA 3015 (EPA, 1996), utilizando-se a técnica de digestão por microondas em sistema fechado (CEM 2000).

A determinação dos metais nos extratos digeridos foi realizada por espectrometria de absorção atômica (Varian) ou espectrometria de emissão atômica com fonte de plasma induzida (Perkin Elmer ICP-AES Optima 3.000).

Os compostos orgânicos voláteis e os hidrocarbonetos policíclicos aromáticos pesquisados em algumas amostras brutas em questão - cujos processos de produção das indústrias geradoras indicavam a presença de tais substâncias - foram determinados por cromatografia gasosa acoplada à espectrometria de massas (CG/EM - Varian), após extração com solventes (EPA, 1996).

\section{Resultados e discussão}

Das 21 amostras analisadas, duas foram classificadas como resíduos perigosos, uma como resíduo inerte e 18 como resíduos não-inertes.

Nenhuma das amostras dos resíduos estudados apresentou valores dos parâmetros analisados acima dos limites descritos no Anexo GListagem no 7 da NBR 10.004 nos extratos lixiviados.

Os resíduos perigosos foram assim classificados pois continham em sua massa bruta compostos orgânicos voláteis e hidrocarbonetos policíclicos aromáticos descritos no Anexo D - 
Listagem no 4 da NBR 10.004 como substâncias que conferem periculosidade aos resíduos. As amostras A e B, originadas do setor petroquímico, foram classificadas como resíduos perigosos por apresentarem as seguintes substâncias: 1,1,1-tricloroetano; benzeno; tricloroeteno; tolueno; tetracloroeteno; naftaleno; fluoranteno; benzo[a] antraceno; criseno; benzo[b]fluoranteno; benzo[a]pireno; indeno[1,2,3-cd]pireno e dibenzo[a,h] antraceno.

A única amostra classificada como resíduo inerte foi originada de uma indústria de beneficiamento de minerais (amostra $U$ ), onde todos os parâmetros analisados apresentaram valores abaixo dos limites descritos no Anexo H - Listagem no 8 da NBR 10.004 para o extrato solubilizado (ABNT, 1987c).

Os resíduos não-inertes receberam especial atenção neste trabalho, uma vez que são passíveis de serem dispostos em aterros controlados e vazadouros de lixo, junto com os resíduos urbanos. Esses resíduos foram assim classificados porque apresentaram um ou mais dos parâmetros analisados em concentrações acima dos limites permitidos pelo Anexo $\mathrm{H}-$
Listagem no 8 da NBR 10.004. Esses limites foram estabelecidos com base nos padrões brasileiros de potabilidade de água vigentes na época da elaboração da NBR 10.004, descritos na Portaria do Ministério da Saúde no 56 de 14/03/77 e que são os mesmos da ainda vigente Portaria do Ministério da Saúde no 36 de 19/01/90 (ABNT, 1987c).

Os parâmetros que contribuíram para a classificação das amostras em resíduos nãoinertes (amostras C a T, originadas dos setores químico, de beneficiamento de minerais, metalúrgico, alimentício e de bebidas) foram os seguintes: cádmio; cianetos; cromo; fenol; fluoretos; nitratos; alumínio; cloretos; cobre; dureza; ferro; manganês; sódio; surfactantes; sulfato e zinco. Na Tabela 1 são descritos os parâmetros analisados em cada amostra de extrato solubilizado, bem como as amostras que apresentaram algum parâmetro em concentrações acima dos valores descritos no Anexo $\mathrm{H}$ - Listagem no 8 .

Dos parâmetros que classificaram as amostras em resíduos não-inertes, os que foram observados com mais freqüência foram: fenol,

Tabela 1

Parâmetros descritos no Anexo H - Listagem no 8 da NBR 10.004 analisados nas amostras estudadas e nas amostras que apresentaram parâmetros em concentrações $(\mathrm{mg} / \mathrm{L})$ acima do limite máximo permitido para o extrato solubilizado.

\begin{tabular}{|c|c|c|}
\hline Parâmetros & Amostras analisadas & Amostras com valores acima do permitido \\
\hline Arsênio & $A, B, D, E, F, G, J, M, N, Q$ & - \\
\hline Bário & $D, E, F, G, I, J, Q$ & - \\
\hline Cádmio & $A, B, D, E, F, G, I, J, P, U$ & $A, B$ \\
\hline Chumbo & $A, B, D, E, F, G, I, J, M, N, P, Q, U$ & - \\
\hline Cianetos & $A, B, D, E, F, G, I, J, N, Q, R$ & $A, B, D, G, I$ \\
\hline Cromo & $A, B, D, E, F, G, I, J, N, P, Q, S, T, U$ & $\mathrm{Q}$ \\
\hline Fenol & $A, B, D, E, F, G, J, M, N, P, Q, R, U$ & $A, B, E, F, G, M, N, Q, R$ \\
\hline Fluoretos & $A, B, C, D, E, F, G, H, I, J, K, N, P, Q$ & $A, B$ \\
\hline Mercúrio & $D, E, F, G, J$ & - \\
\hline Nitrato & $A, B, C, D, E, F, G, H, I, J, K, L, N, O, R, \cup$ & $A, B, G, O, R$ \\
\hline Prata & $D, E, F, G, J$ & - \\
\hline Selênio & $D, E, F, G, J$ & - \\
\hline Alumínio & $A, B, D, E, F, G, I, J, K, M, N, O, Q, S, T, U$ & $D, G, I, J, M, N, O, Q, S, T$ \\
\hline Cloretos & $A, B, C, D, E, F, G, H, I, J, K, L, M, N, O, Q, U$ & $A, B, L$ \\
\hline Cobre & $A, B, D, E, F, G, I, J, M, P, Q, S, T, U$ & $\mathrm{G}, \mathrm{Q}$ \\
\hline Dureza & $A, B, C, D, E, F, G, H, I, J, K, L, M, N, O, Q, R, S, T, U$ & $A, B, F, M, N, R$ \\
\hline Ferro & $A, B, D, E, F, G, J, L, M, N, O, P, Q, R, S, T, U$ & $A, B, D, E, F, J, L, M, N, O, Q, R$ \\
\hline Manganês & $A, B, D, E, F, G, J, N, S, T, U$ & $A, B, D, E, F, J$ \\
\hline Sódio & $A, B, C, D, E, F, G, I, J, K, L, M, N, O, R, U$ & $A, B, G, K, R$ \\
\hline Surfactantes & $A, B, C, D, E, F, G, H, I, J, K, L, M, N, O, P, Q, R, \cup$ & $A, B, C, D, E, F, G, H, K, M, O, P, Q, R$ \\
\hline Sulfato & $A, B, C, D, E, F, G, H, I, J, K, L, M, N, O, P, Q, U$ & $A, B$ \\
\hline Zinco & $A, B, D, E, F, G, I, J, M, N, P, Q, R, U$ & $\mathrm{Q}, \mathrm{R}$ \\
\hline
\end{tabular}


alumínio, ferro, manganês e surfactantes. O fenol foi encontrado em concentrações acima do permitido em nove amostras; o alumínio, em dez delas; o ferro, em 12; o manganês, em seis; e surfactantes, em 14 das 18 amostras de resíduos não-inertes (Tabela 1). Na Tabela 2 são descritas as faixas de concentração encontradas desses contaminantes (os limites de detecção de alumínio, ferro e manganês relatados referem-se à técnica de ICP-AES) e o limite máximo estabelecido pela NBR 10.004 para o extrato solubilizado.

De todos os parâmetros que apresentaram concentrações superiores às recomendadas para o extrato solubilizado, podem ser destacados o fenol, o alumínio e o manganês, por suas características tóxicas. Algumas amostras apresentaram concentrações de alumínio quase 300 vezes maiores do que o limite estabelecido, enquanto no caso do manganês foi encontrada uma amostra com quase 50 vezes o limite. A maior concentração de fenol encontrada foi 100 vezes maior do que o valor máximo permitido.

A contaminação por fenol de águas usadas para consumo humano pode levar ao aumento da incidência de distúrbios gastrointestinais (Richardson \& Gangolli, 1992). Já a presença de alumínio e manganês na água merece atenção porque esses contaminantes estão relacionados ao desenvolvimento de doenças degenerativas do sistema nervoso central (Goyer, 1995; Richardson \& Gangolli, 1992).

As concentrações elevadas de alumínio e ferro encontradas nos resíduos analisados (principalmente os lodos de estação de tratamento de efluentes e águas) podem ter origem nos sulfatos e cloretos de alumínio e ferro, comumente utilizados nos processos de tratamento de águas e efluentes líquidos industriais (Braile \& Cavalcanti, 1993).

As elevadas concentrações de surfactantes encontradas - substâncias tensoativas que reagem ao azul de metileno - provavelmente foram originadas da grande variedade de detergentes utilizados para limpeza em diversos setores dos processos industriais (Shreve \& Brink, 1997).

O fenol identificado nas amostras pode ser oriundo de várias fontes, pois compostos fenólicos são usados como desinfetantes e coadjuvantes em vários segmentos industriais (Richardson \& Gangolli, 1992; Shreve \& Brink, 1997).

\section{Conclusões}

Resíduos industriais não-inertes não deveriam ser depositados livremente em aterros contro-

\begin{tabular}{|c|c|c|}
\hline \multicolumn{3}{|c|}{$\begin{array}{l}\text { Faixas de concentração de alumínio, ferro, manganês, fenol } \\
\text { e surfactantes encontradas nas amostras estudadas e limites máximos } \\
\text { descritos no Anexo H - Listagem no } 8 \text { da NBR } 10.004 \text { para o extrato solubilizado. }\end{array}$} \\
\hline Parâmetro analisado & $\begin{array}{l}\text { Faixas de concentração } \\
\qquad(\mathrm{mg} / \mathrm{L})\end{array}$ & $\begin{array}{l}\text { Limite máximo no extrato } \\
\qquad(\mathrm{mg} / \mathrm{L})\end{array}$ \\
\hline Alumínio & $<0,010-57,0$ & 0,2 \\
\hline Ferro & $<0,006-59,0$ & 0,3 \\
\hline Manganês & $<0,003-4,6$ & 0,1 \\
\hline Fenol & $<0,001-0,12$ & 0,001 \\
\hline Surfactantes & $<0,01-2,4$ & 0,2 \\
\hline
\end{tabular}

Fonte dos limites máximos: NBR 10.004 - Anexo H - Listagem no 8 - Padrões para o teste de solubilização (ABNT, 1987c).

lados sem os cuidados necessários. Esses resíduos apresentam propriedades que podem comprometer a área onde o aterro está localizado e áreas sob sua influência, como combustibilidade, biodegradabilidade ou solubilidade em água (ABNT, 1987c).

Dessa forma, muitas indústrias, apesar de procederem de forma correta na classificação e declaração ao órgão ambiental dos resíduos gerados em seu processo de produção, ao destinarem esses resíduos a um aterro municipal muitas vezes com autorização do órgão fiscalizador -, estão contribuindo para o agravamento do quadro de degradação ambiental observado nas áreas de disposição de resíduos encontradas no Estado do Rio de Janeiro, e colocando em risco todos os benefícios almejados por meio da certificação ISO 14.000.

Uma vez que os aterros controlados e vazadouros não possuem infra-estrutura sanitária adequada para evitar os problemas oriundos do despejo de toneladas de resíduos urbanos, a disposição de resíduos sólidos industriais nãoinertes nessas áreas está contribuindo para que as várias substâncias químicas de interesse toxicológico encontradas nos resíduos possam ser acumuladas ou exportadas a partir desses aterros, agravando ainda mais a situação atualmente observada.

É desejável e necessário que a preocupação com o gerenciamento adequado dos resíduos industriais seja seriamente considerada por todas as esferas envolvidas, inclusive pelo setor normativo e fiscalizador. Assim sendo, é primordial o desenvolvimento de uma política de gerenciamento de resíduos para o Estado do Rio de Janeiro que garanta opções de destinação e tratamento geograficamente próximas e economicamente viáveis tanto para os resíduos ur- 
banos como para os resíduos industriais, a fim de permitir que ações integradas proporcionem a melhoria da situação ora vigente e a diminuição dos riscos associados ao destino inadequado dos resíduos sólidos.

\section{Agradecimentos}

A autora gostaria de expressar seus agradecimentos ao Prof. Dr. Annibal Duarte Pereira Netto, da Universidade Federal Fluminense; aos profissionais do Centro de Tecnologia Ambiental da Federação das Indústrias do Estado do Rio de Janeiro e a Ana Paula Fittipaldi, da Fundação Estadual de Engenharia do Meio Ambiente.

\section{Referências}

ABNT (Associação Brasileira de Normas Técnicas), 1987a. Amostragem de Resíduos - Procedimento NBR 10.007. Rio de Janeiro: ABNT.

ABNT (Associação Brasileira de Normas Técnicas), 1987b. Lixiviação de Resíduos - Procedimento NBR 10.005. Rio de Janeiro: ABNT.

ABNT (Associação Brasileira de Normas Técnicas), 1987c. Resíduos Sólidos - Classificação - NBR 10.004. Rio de Janeiro: ABNT.

ABNT (Associação Brasileira de Normas Técnicas), 1987d. Solubilização de Resíduos - Procedimento - NBR 10.006. Rio de Janeiro: ABNT.

ABNT (Associação Brasileira de Normas Técnicas), 1996. Sistema de Gestão Ambiental - Diretrizes Gerais sobre os Princípios, Sistemas e Técnicas de Apoio - NBR ISO 14.004. Rio de Janeiro: ABNT.

APHA (American Public Health Association), 1995. Standard Methods for the Examination of Water and Wastewater. Washington, DC: APHA.

BRAILE, P. M. \& CAVALCANTI, J. E. W. A., 1993. Manual de Tratamento de Águas Residuárias Industriais. São Paulo: Companhia de Tecnologia de Saneamento Ambiental.

COMLURB (Companhia Municipal de Limpeza Urbana), 1993. Projeto de Recuperação do Aterro Metropolitano de Gramacho. Rio de Janeiro: COMLURB. (mimeo.)

EPA (Environmental Protection Agency), 1996. Test Methods for Evaluating Solid Waste Physical/ Chemical Methods - SW 846. Richmond: EPA/National Technical Information Service.

FEEMA (Fundação Estadual de Engenharia do Meio Ambiente), 1998. Sem reciclagem o lixo ainda atormenta e reduz qualidade de vida. Revista FEEMA, 20:34-40.

FEEMA (Fundação Estadual de Engenharia do Meio Ambiente), 2000. Gestão de Resíduos - Relatório Semestral de Atividades do Programa de Despoluição da Baía de Guanabara - Setembro/2000. Rio de Janeiro: FEEMA. (mimeo.)
GOYER, R. A., 1995. Toxic effects of metals. In: Casarett and Doull's Toxicology: The Basic Science of Poisons (C. D. Klaassen, ed.), pp. 691-736, New York: McGraw-Hill Companies.

PEREIRA NETTO, A. D.; SISINNO, C. L. S.; MOREIRA, J. C. \& ARBILLA, G., 2002. Polycyclic aromatic hydrocarbons in leachate from a municipal solid waste dump of Niterói city, RJ, Brazil. Bulletin of Environmental Contamination and Toxicology, 68:148-154.

RICHARDSON, M. L. \& GANGOLLI, S., 1992. The Dictionary of Substances and their Effects. Northamptonshire: Royal Society of Chemistry.

SHREVE, R. N. \& BRINK Jr., J. A., 1997. Indústrias de Processos Químicos. Rio de Janeiro: Editora Guanabara Koogan.

SISINNO, C. L. S. \& MOREIRA, J. C., 1996. Avaliação da contaminação e poluição ambiental na área de influência do aterro controlado do Morro do Céu, Niterói, Brasil. Cadernos de Saúde Pública, 12: 515-523.

SISINNO, C. L. S. \& OLIVEIRA, R. M., 2000. Impacto ambiental de grandes depósitos de resíduos sólidos urbanos e industriais. In: Resíduos Sólidos, Ambiente e Saúde: Uma Visão Multidisciplinar (C. L. S. Sisinno \& R. M. Oliveira, org.), pp. 41-57, Rio de Janeiro: Editora Fiocruz.

USA (United States of America), 1994. Code of Federal Registry. Title 40 - Protection of Environment Parts 260 to 299. Washington, DC: U.S. Government Printing Office.

Recebido em 26 de fevereiro de 2002

Versão final reapresentada em 11 de junho de 2002 Aprovado em 19 de agosto de 2002 